\title{
Highly sensitive detection of low abundance BRAF V600E mutation in fine-needle aspiration samples by Zip recombinase polymerase amplification
}

Lutan Zhang, ${ }^{\mathrm{a}, \mathrm{c}, 1}$, Jian Peng ${ }^{\mathrm{a}, 1}$, Junman Chen ${ }^{\mathrm{b}, 1}$, Lulu Xu ${ }^{\mathrm{a}}$, Yangli Zhanga ${ }^{\mathrm{a}}$, Ying Li ${ }^{\mathrm{a}}$, Jie Zhao $^{\text {a }}$, Linguo Xiang ${ }^{\mathrm{a}}$, Yunsheng Ge ${ }^{* \mathrm{c}}$, Wei Cheng*a

a The Center for Clinical Molecular Medical detection, The First Affiliated Hospital of Chongqing Medical University, Chongqing 400016, P.R. China

${ }^{b}$ Key Laboratory of Clinical Laboratory Diagnostics (Ministry of Education), College of Laboratory Medicine, Chongqing Medical University, Chongqing 400016, P.R.

China

c United Diagnostic and Research Center for Clinical Genetics, Women and Children's Hospital, School of Medicine \& School of Public Health, Xiamen University, Xiamen 361000, People's Republic of China

${ }^{*}$ Corresponding author at: Tel.: +86-23-89011713.

E-mail address: chengwei@hospital.cqmu.edu.cn (W. Cheng). 
Table of contents

Reagents and Apparatus-----------------------------------------------------------------------S-3

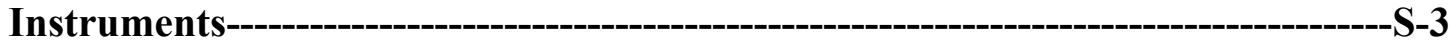

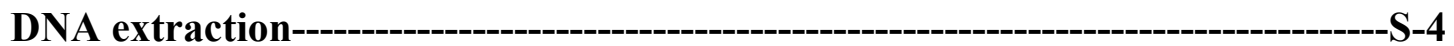

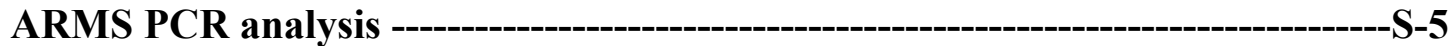

Performance comparison of phi 29 polymerase and klenow Fragment (3'-5' exo-)

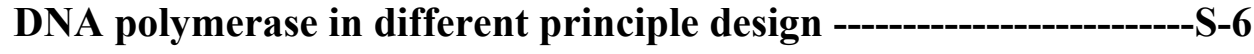

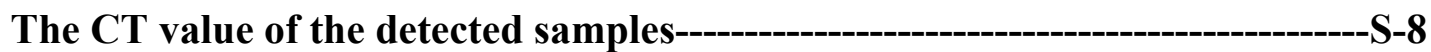




\section{Reagents and materials}

All oligonucleotides except ZNA were synthesized and HPLC puried by Sangon

Biotechnology Co. Ltd. (Shanghai, China). TwistAmp Basic RPA kit was gotten from TwistDX (UK). ZNA probe was synthesized and puried by DAAN Gene Co. Ltd. (Zhongshan, China). Phi 29 DNA polymerase was obtained from ThermoFisher Scientific Co. Ltd. (Massachusetts, U.S.A.). SYBR Green I was purchased from Solarbio Life Sciences (Beijing, China). All of the sequences are listed in Table S1.

Table S1. Sequences of targets and probes used in this assay.

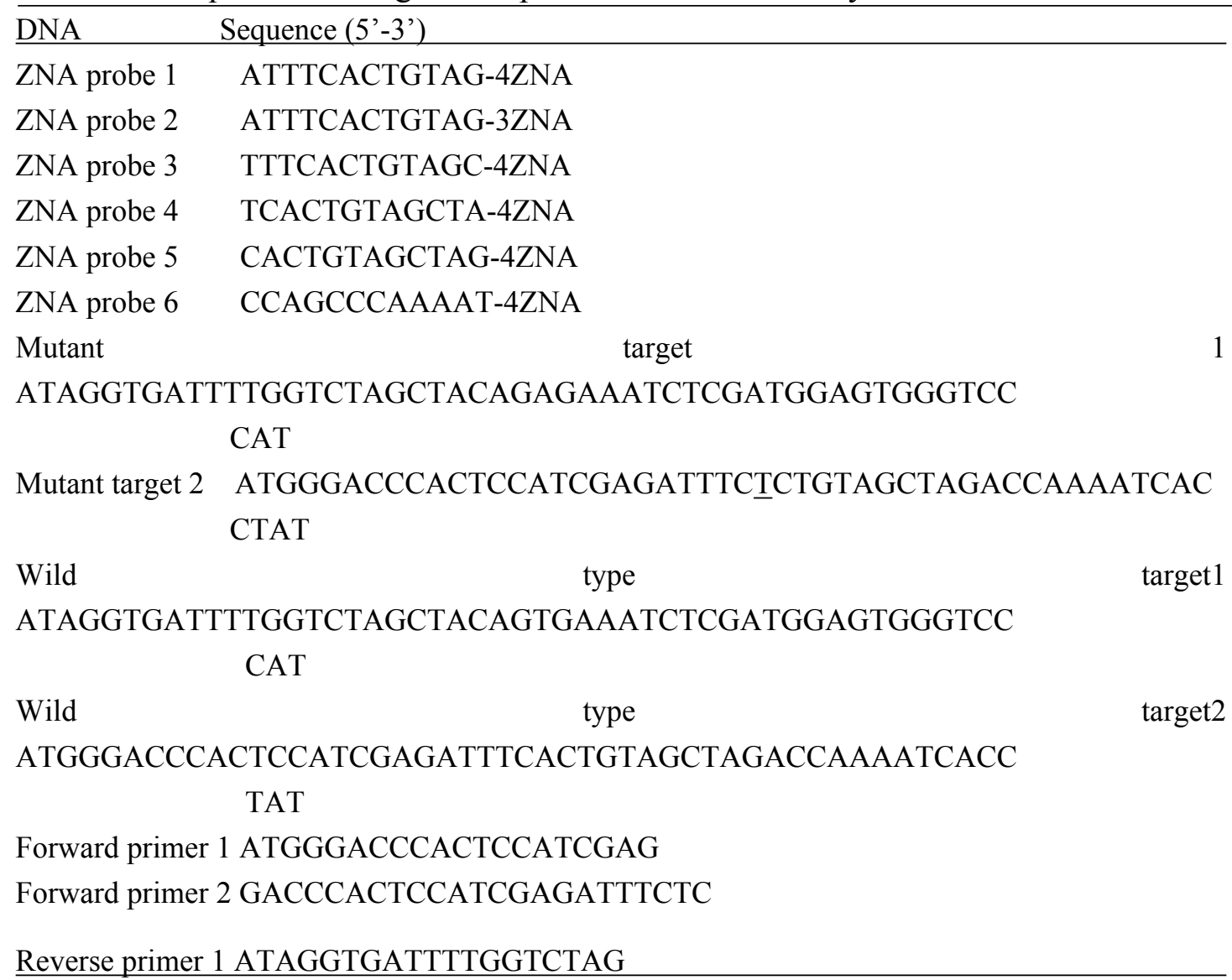

\section{Instruments}


Rapid high-throughput, plate-based real-time PCR amplification and detection instruments were carried on LightCycler ${ }^{\circledR} 480$ Instrument II (Rothe, Co., Ltd., Germany) and Applied Biosystems ${ }^{\mathrm{TM}} 7500$ (Thermo Fisher Scientific, Co., Ltd., Shanghai, China). The gel electrophoresis and gel image were performed on DYY-6C electrophoresis analyzer (Liuyi Instrument Company, China) and Bio-rad ChemDoc XRS (Bio-Rad, USA), respectively. Automate low-throughput sample preparation was used by Auto-Pure32A Nucleic Acid Purification System (Allsheng Co., Ltd., Hangzhou, China). The DNA concentrations were quantified according to the absorption at $260 \mathrm{~nm}$ with a NanoDrop One Microvolume UV-Vis Spectrophotometer (Thermo Fisher Scientific, Co., Ltd., Shanghai, China).

\section{DNA extraction}

\section{AmoyDx@FFPE DNA Kit}

$180 \mu \mathrm{L}$ Buffer DTL and $20 \mu \mathrm{L}$ proteinase $\mathrm{K}$ solution were added in samples, and heated up to $56^{\circ} \mathrm{C}$ for 1 hour after shaking and mixing. Next, $10 \mu \mathrm{L}$ Buffer DES were added into the mixture and heat for 1 hour in constant temperature incubator. Then, $200 \mu \mathrm{L}$ Buffer DTB and $200 \mu \mathrm{L}$ absolute ethyl alcohol were mixed with the solution and transferred the solution into a DNA adsorption column followed by centrifuging (10000×g $1 \mathrm{~min})$. After discarding filtrate, $600 \mu \mathrm{L}$ Buffer DW1 was added into adsorption column to wash by centrifuging. Repeat the washing step by using $600 \mu \mathrm{L}$ Buffer DW2. The DNA was eluted in $100 \mu \mathrm{L}$ Buffer DTE by centrifuging at $13000 \mathrm{~g}$ for 1 minute. For detailed protocol of AmoyDx@FFPE DNA Kit, please visit 
http://www.amoydx.com/productDetail_30.html.

\section{BloodGen Mini Kit}

1. Sample treatment: For $200 \mathrm{ul}$ of sample, add the sample to the centrifuge tube (self-prepared) and proceed directly to the next step.

2. Add 20 ul Proteinase K to above solution and vortex to mix thoroughly.

3. Add 200 ul Buffer GL, then vortex to mix thoroughly.

4. Incubate at $56^{\circ} \mathrm{C}$ water bath for 10 minutes, inverting the tube upside down serval times internally to mix well.

5. Add 200 ul $100 \%$ ethanol and invert the tube upside down several times. Centrifuge briefly to collect all the liquid to the bottom of the tube.

6. Transfer the solution obtained from step 5 to a column with collection tube (Spin Columns DM); Transfer multiple times if needed; Centrifuge at 12,000 rpm for 1 minute; Discard the waste in the collection tube and put the column back to the collection tube.

7. Add $500 \mu \mathrm{l}$ Buffer GW1 to the column (check if $100 \%$ ethanol has been added before use). Centrifuge for 1 minute at $12,000 \mathrm{rpm}$. Discard the waste from the collection tube and put back the column to the collection tube.

8. Add $500 \mu$ Buffer GW2 to the column (check if $100 \%$ ethanol has been added before use). Centrifuge for 1 minute at $12,000 \mathrm{rpm}$. Discard the waste from the collection tube and put back the column to the collection tube.

9. Centrifuge at $12,000 \mathrm{rpm}$ for 2 minutes and discard the waste from the 
collection tube. Leave the column at room temperature to dry thoroughly.

10. Place the column in a new $1.5 \mathrm{ml}$ centrifuge tube (self-prepared) and add $50-200 \mu 1$ of Buffer GE or autoclaved $\mathrm{H} 2 \mathrm{O}$ in the middle of the membrane and allow it to stand at room temperature for 2-5 minutes. Centrifuge at $12,000 \mathrm{rpm}$ for 1 minute and collect the DNA solution. Store the DNA at $-20^{\circ} \mathrm{C}$.

For detailed protocol of BloodGen Mini Kit, please visit https://www.cwbiosciences.com/goods/index/id/26.

\section{Performance comparison of phi 29 polymerase and klenow fragment ( $3^{\prime}-5$ ' exo-) DNA polymerase in different principle design}

In this research, different designs of Z-RPA assay were explored and tested in terms of feasibility. As shown in Figure 2A, the first design contains the competitive hybridization between 3' end of forward primer and ZNA probe, where single base mismatch makes ZNA-mutant target unstable thus letting complementary hybridization of primer and mutant target. In the second design, where primer doesn't overlap ZNA, ZNA probes were unwound under the elongation reaction by polymerases when mutant type targets existed. As Figure S1A shown, in the test of first principle design, the reaction run with klenow fragment (3'-5' exo-) DNA polymerase had no product band output, demonstrating that ZNA probes can bond target DNA more steadily than DNA primers, and DNA primers were not able to displace ZNA to start RPA reaction. While the same electrophoresis results was obtained by detecting mutation target in the reaction with phi 29 polymerase (Figure S1B), but the results of fluorescence signals were different, indicating that the 
generation of by-products were few in the competitive hybridization principle design by using phi 29 polymerase, which verifyed the possibility that the inherent $3^{\prime}-5^{\prime}$ proofreading exonuclease activity of phi 29 polymerase made reaction start through $3^{\prime}-5^{\prime}$ exonucleolysis of $3^{\prime}$ end protruding primer, rather than the competitive displacement of primers. On the other hand, in the measurement of elongation blocking principle design, products band was observed upon detection of mutant target by Z-RPA with either phi 29 polymerase or klenow fragment (3'-5' exo-) DNA polymerase (Figure 1A, Figure S1A). The consistent detection results proved the feasibility of the second design program, and the ZNA probes can only be destabilized by force of the powerful strand displacement property of polymerase. And the similar performance of the two kinds of polymerases were testified in RPA system (Figure S2). Therefore, the second principle design and Phi 29 polymerase was selected to apply to our research.

(A)

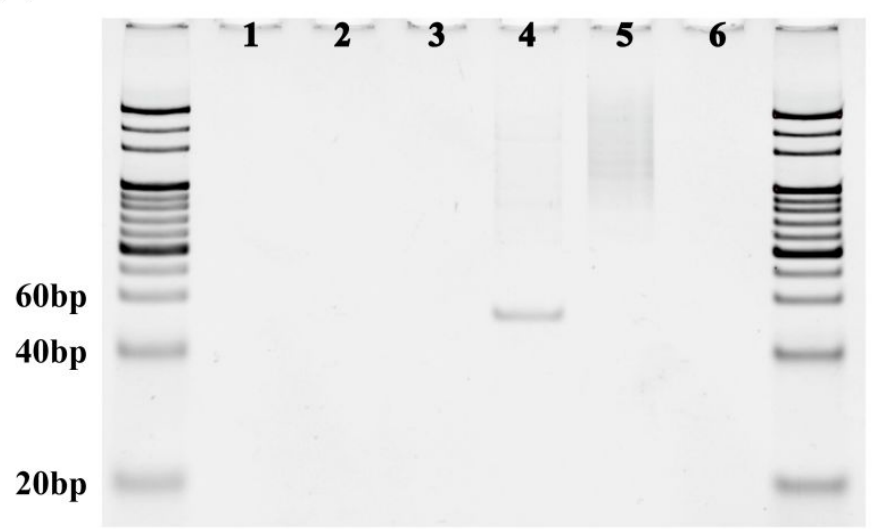

(B)

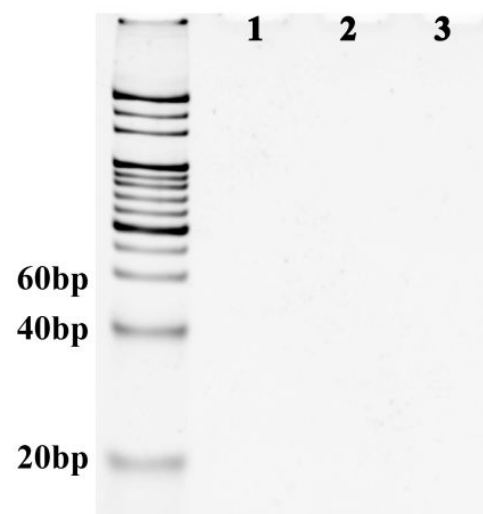

Figure S1. (A) Polyacrylamide gel electrophoresis of the products of Z-RPA for the mutant targets (Lane 1, 4), wild targets (Lane 2,5) and blank controls (Lane 3,6) with klenow fragment (3'-5' exo-) DNA polymerase under competitive hybridization (Lanes 1-3) and elongation blocking (Lanes 4-6) principle design. (B) Polyacrylamide gel electrophoresis of the products of Z-RPA for the mutant targets (Lane 1), wild targets (Lane 2) and blank controls (Lane 3) with phi 29 polymerase under competitive hybridization principle design. 


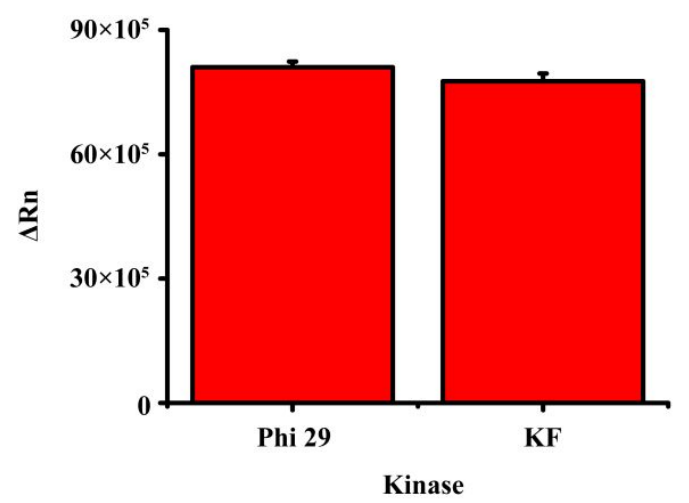

Figure S2. The comparison of the performance between phi 29 DNA polymerase and klenow fragment (3'-5' exo-) DNA polymerase.

\section{The CT value of the detected samples}

Table S2. The CT value of the detected samples by Z-RPA.

\begin{tabular}{|c|c|c|c|c|c|}
\hline Number & Sample code & $\mathrm{CT}$ value (Malignant) & Number & Sample code & CT value (Benign) \\
\hline 1 & 042504 & 14.64 & 1 & 042501 & Undetermined \\
\hline 2 & 042505 & 25.29 & 2 & 042502 & Undetermined \\
\hline 3 & 042509 & 20.83 & 3 & 042506 & Undetermined \\
\hline 4 & 042512 & 13.04 & 4 & 042510 & Undetermined \\
\hline 5 & 043002 & 9.88 & 5 & 042514 & Undetermined \\
\hline 6 & 043006 & 7.43 & 6 & 042517 & Undetermined \\
\hline 7 & 043011 & 12.13 & 7 & 053152 & Undetermined \\
\hline 8 & 050710 & 13.96 & 8 & 062502 & Undetermined \\
\hline 9 & 051612 & 19.34 & 9 & 062503 & Undetermined \\
\hline 10 & 051615 & 13.60 & 10 & 062504 & Undetermined \\
\hline 11 & 053004 & 10.00 & 11 & 062506 & Undetermined \\
\hline 12 & 062501 & 23.75 & 12 & 062507 & Undetermined \\
\hline 13 & 062508 & 13.17 & 13 & 062511 & Undetermined \\
\hline 14 & 062510 & 25.01 & 14 & 062514 & Undetermined \\
\hline 15 & 062512 & 14.14 & 15 & 062515 & Undetermined \\
\hline 16 & 062513 & 23.41 & 16 & 062527 & Undetermined \\
\hline 17 & 062518 & 22.72 & 17 & 062528 & Undetermined \\
\hline 18 & 062519 & 23.08 & 18 & 062529 & Undetermined \\
\hline 19 & 062520 & 19.89 & 19 & 062530 & Undetermined \\
\hline 20 & 062521 & 15.50 & 20 & 062570 & Undetermined \\
\hline 21 & 062523 & 19.04 & 21 & 062580 & Undetermined \\
\hline 22 & 062524 & 24.16 & 22 & 062583 & Undetermined \\
\hline 23 & 062525 & 24.40 & & & \\
\hline 24 & 062581 & 10.98 & & & \\
\hline 25 & 062598 & 17.77 & & & \\
\hline 26 & 0625145 & 12.43 & & & \\
\hline
\end{tabular}


